\title{
A New Quasi Poison-Lindley Distribution: Properties and Applications
}

\author{
Munindra Borah \\ Department of Mathematical Sciences \\ Tezpur University, Napaam, Assam, India-784028 \\ mborah@tezu.ernet.in \\ Junali Hazarika* \\ Department of Mathematical Sciences \\ Tezpur University, Napaam, Assam, India-784028 \\ junali@tezu.ernet.in
}

Received 6 April 2016

Accepted 10 May 2017

\begin{abstract}
A new two parameter quasi Poisson Lindley (NQPL) distribution is derived by using the general approach of compounding. One parameter Lindley distribution and geometric distribution may be obtained as a particular case. The new distribution is shown to be unimodal and overdispersed. A few distributional properties and recurrence relation of the proposed distribution are examined. The size biased new quasi Poisson Lindley (SBNQPL) distribution is also discussed. The method of moments and method of maximum likelihood have been used for estimating the parameters for both NQPL and SBNQPL distributions have been fitted to some real data sets for testing its goodness of fit and found to perform better than other basic distributions.
\end{abstract}

Keywords: Unimodality, Overdispersion, Method of Moments and Method of Maximum Likelihood estimation.

2000 Mathematics Subject Classification: 22E46, 53C35, 57S20.

\section{Introduction}

Lindley [9] introduced a one-parameter continuous distribution, known as Lindley distribution, given by its probability density function (pdf)

$$
f(x ; \theta)=\frac{\theta^{2}(1+x)}{\theta+1} e^{-\theta x}, x>0, \theta>0
$$

Lindley distribution is a mixture of exponential $(\theta)$ and gamma $(2, \theta)$ distributions.

The one parameter Poisson Lindley (PL) distribution is given by the probability mass function (pmf)

$$
P_{1}(x ; \theta)=\frac{\theta^{2}(x+\theta+2)}{(1+\theta)^{x+3}}, \quad x=0,1, \ldots \ldots, \theta>0
$$

was introduced by Sankaran [10] to model count data. Because of having only one parameter, the PoissonLindley distribution does not provide enough flexibility for analyzing different types of lifetime data. To increase the flexibility for modeling purposes it will be useful to consider further alternatives of this

\footnotetext{
${ }^{*}$ Corresponding author.
} 
distribution. Ghitany and Al-Mutairi [7] discussed estimation methods for the discrete Poisson-Lindley distribution.

Shanker and Mishra [11] introduced a quasi Lindley distribution (QLD) with parameters $\theta$ and $\alpha$ defined by its probability density function (pdf)

$$
f(x ; \alpha, \theta)=\frac{\theta(\alpha+\theta x)}{1+\alpha} e^{-\theta x} ; \quad x>0, \theta>0, \alpha>-1
$$

Shanker and Mishra [12] obtained a quasi Poisson-Lindley (QPL) distribution given by its probability mass function (pmf)

$$
P_{2}(x ; \theta, \alpha)=\frac{\theta}{(\alpha+1)(1+\theta)^{x+2}}\left(\alpha+\frac{\theta(x+1)}{\theta+1}\right), x=0,1, \ldots, \alpha>-1
$$

It can be seen that (1.2) is a particular case of (1.4) at $\alpha=\theta$ and at $\alpha=0$, it reduces to the gamma distribution with parameters $(2, \theta)$. Shanker and Mishra [12] have shown that (1.4) is a better model than the PL distribution of Sankaran [10] for modeling waiting and survival times data.

The two parameter New Quasi Lindley (NQL) distribution is introduced by Shanker and Ghebretsadik [13] with pdf

$$
f(x)=\frac{\theta^{2}(\theta+\alpha x)}{\theta^{2}+\alpha} e^{-\theta x} ; \quad x>0, \theta>0, \alpha<-\theta^{2}
$$

It has been obtained that Lindley and exponential distribution are the particular case of (1.5) at $\alpha=\theta$ and $\alpha=0$. Ghitany and Al-Mutairi discussed estimation methods for discrete Poisson-Lindley distribution where the parameters are estimated by using the method of moments (MoM) and maximum likelihood (ML). They have examined the un-biasedness, uniqueness and asymptotic properties of the distribution.

In this paper, a New quasi Poisson-Lindley distribution is proposed by compounding Poisson distribution with that of new quasi Lindley distribution. In section 2, a new quasi Poisson Lindley distribution is defined and discussed its basic properties including the behavior of the probability mass function. Distributional properties and generating functions are discussed in section 3 . In section 4, probability generating function is obtained. Size biased version of the proposed distribution is obtained with its generating function and recurrence relation is obtained in section 5. In section 6 and 7, Method of moment and method of maximum likelihood have been discussed for estimating the parameters.

\section{New Quasi Poisson-Lindley (NQPL) Distribution}

Suppose the parameter $\lambda$ of the Poisson distribution follows the new quasi Lindley distribution (5), then the compounded Poisson distribution is given as

$$
\begin{aligned}
P_{3}(x ; \theta, \alpha) & =\int_{0}^{\infty} \frac{e^{-\lambda} \lambda^{x}}{x !} \frac{\theta^{2}}{\theta^{2}+\alpha}(\theta+\alpha \lambda) e^{-\theta \lambda} d \lambda \\
& =\frac{\theta^{2}}{(1+\theta)^{x+2}}\left(1+\frac{\theta+\alpha x}{\theta^{2}+\alpha}\right), \quad x=0,1, \ldots,
\end{aligned}
$$

where $\theta>0$ and $\alpha>\max \left(-\frac{\theta(1+\theta)}{1+n},-\theta^{2}\right)$ or $\alpha<\min \left(-\frac{\theta(1+\theta)}{1+n},-\theta^{2}\right)$.

\section{ParticularCases:}

(i) For $\alpha=\theta,(2.1)$ reduces to one parameter Poisson Lindley distribution.

(ii) For $\alpha=0,(2.1)$ reduces to geometric distribution with probability $\frac{\theta}{1+\theta}$. 

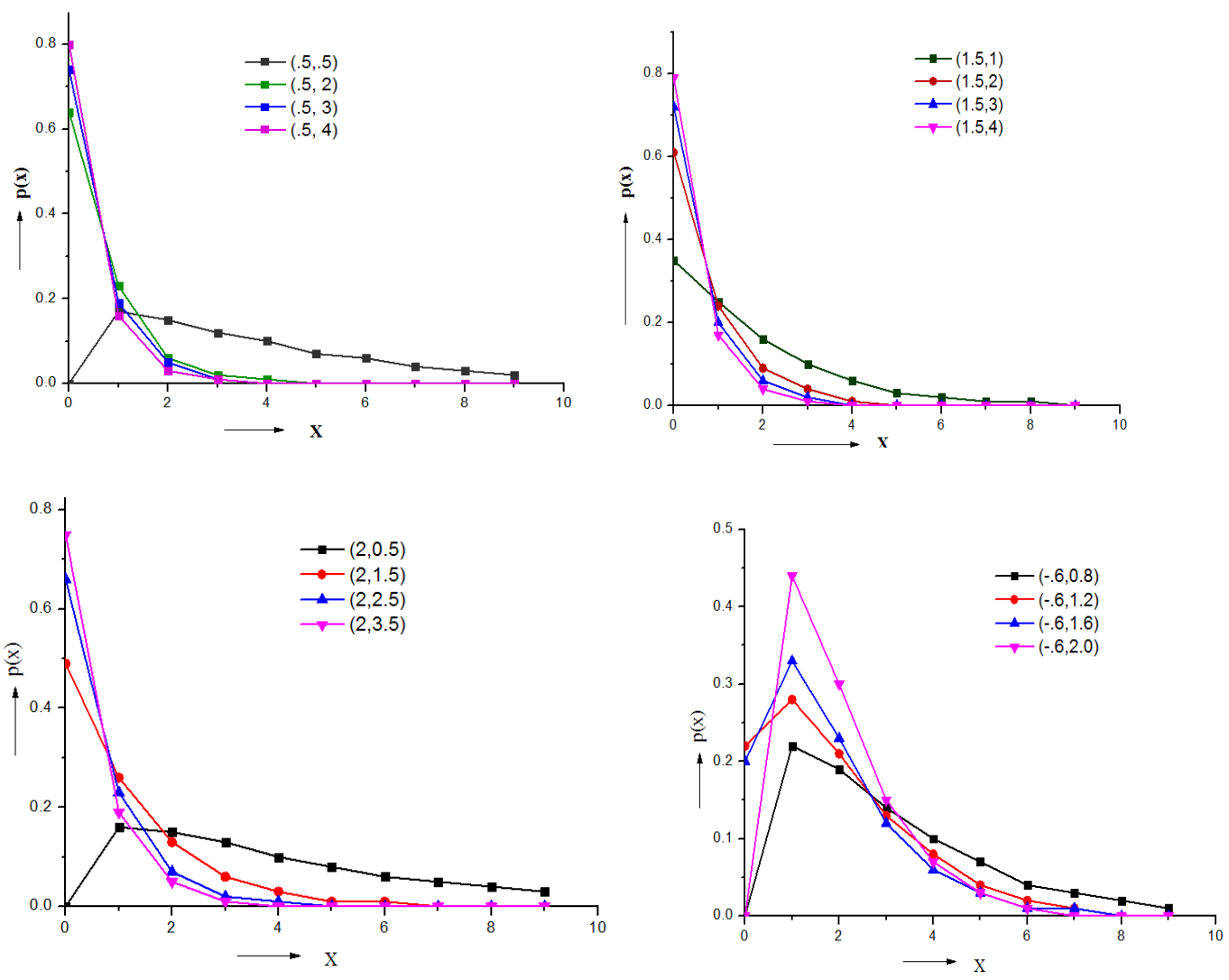

Figure 1. Plot of NQPL distribution for different values of parameters $\alpha$ and $\theta$.

\section{Distributional properties:}

\subsection{Shape of the probability function}

It can be seen that

$$
\frac{P(x+1 ; \theta, \alpha)}{P(x ; \theta, \alpha)}=\frac{1}{1+\theta}\left\{1+\frac{\alpha}{\theta^{2}+\theta+\alpha+\alpha x}\right\}
$$

is a decreasing function in $x ; P(x ; \theta, \alpha)$ is log-concave. Therefore, the new quasi Poisson-Lindley (2.1) distribution is unimodal.

\subsection{Moments, Skewness and Kurtosis}

The $r^{\text {th }}$ factorial moment about origin is obtained as

$$
\mu_{(r)}^{\prime}=E\left[E\left(X^{(r)} \mid \lambda\right)\right],
$$

where, $X^{(r)}=X(X-1) \ldots \ldots(X-r+1)$. 
Hence,

$$
\begin{aligned}
\mu_{(r)}^{\prime} & =\int_{0}^{\infty}\left\{\sum_{x=0}^{\infty} x^{(r)} \frac{e^{-\lambda} \lambda^{x}}{x !}\right\} \frac{\theta^{2}}{\theta^{2}+\alpha}(\theta+\alpha \lambda) e^{-\theta \lambda} d \lambda \\
& =\int_{0}^{\infty} \lambda^{r}\left\{\sum_{x=r}^{\infty} \frac{e^{-\lambda} \lambda^{x-r}}{(x-r) !}\right\} \frac{\theta^{2}}{\theta^{2}+\alpha}(\theta+\alpha \lambda) e^{-\theta \lambda} d \lambda
\end{aligned}
$$

Taking $(x+r)=x$ in (3.1), we have

$$
\mu_{(r)}^{\prime}=\int_{0}^{\infty} \lambda^{r}\left\{\sum_{x=0}^{\infty} \frac{e^{-\lambda} \lambda^{x}}{x !}\right\} \frac{\theta^{2}}{\theta^{2}+\alpha}(\theta+\alpha \lambda) e^{-\theta \lambda} d \lambda
$$

The expression within the bracket is equal to unity which is the sum of the pmf of Poisson distribution. Therefore,

$$
\mu_{(r)}^{\prime}=\frac{\theta^{2}}{\theta^{2}+\alpha} \int_{0}^{\infty} \lambda^{r}(\theta+\alpha \lambda) e^{-\theta \lambda} d \lambda
$$

Now using gamma integral and on simplification we get the $r^{\text {th }}$ factorial moment as

The $r^{\text {th }}$ raw moment about origin is obtained as

$$
\mu_{(r)}^{\prime}=\frac{\Gamma(\mathrm{r}+1)\left\{\theta^{2}+\alpha(r+1)\right\}}{\theta^{r}\left(\theta^{2}+\alpha\right)}, \mathrm{r}=1,2, \ldots, \theta>0 .
$$

$$
\begin{gathered}
\mu_{r}^{\prime}=E\left[E\left(X^{r} \mid \lambda\right)\right] \\
\mu_{r}^{\prime}=\int_{0}^{\infty}\left\{\sum_{x=0}^{\infty} x^{r} \frac{e^{-\lambda} \lambda^{x}}{x !}\right\} \frac{\theta^{2}}{\theta^{2}+\alpha}(\theta+\alpha \lambda) e^{-\theta \lambda} d \lambda
\end{gathered}
$$

The expression in the bracket is the $r^{\text {th }}$ order moment about origin of the Poisson distribution. Taking $r=1$, in equation (3.1) and using the mean of the Poisson distribution we can get the first order raw moment i.e. mean of new quasi Poisson Lindley (NQPL) distribution as

$$
\mu_{1}^{\prime}=\int_{0}^{\infty} \lambda \frac{\theta^{2}}{\theta^{2}+\alpha}(\theta+\alpha \lambda) e^{-\theta \lambda} d \lambda=\frac{\theta^{2}+2 \alpha}{\theta\left(\theta^{2}+\alpha\right)}
$$

Taking $r=2$, in equation (3.1) and using the second order moment of the Poisson distribution we can get the second order moment of new quasi Poisson Lindley distribution as

$$
\mu_{2}^{\prime}=\int_{0}^{\infty}\left(\lambda^{2}+\lambda\right) \frac{\theta^{2}}{\theta^{2}+\alpha}(\theta+\alpha \lambda) e^{-\theta \lambda} d \lambda=\frac{\theta^{2}+2 \alpha}{\theta\left(\theta^{2}+\alpha\right)}+\frac{2\left(\theta^{2}+3 \alpha\right)}{\theta^{2}\left(\theta^{2}+\alpha\right)}
$$

Taking $r=3,4$ in equation (3.1) and using the respective moment of the Poisson distribution we can get the third and fourth order moment of new quasi Poisson Lindley distribution as

$$
\mu^{\prime}{ }_{3}=\frac{\theta^{2}+2 \alpha}{\theta\left(\theta^{2}+\alpha\right)}+\frac{6\left(\theta^{2}+3 \alpha\right)}{\theta^{2}\left(\theta^{2}+\alpha\right)}+\frac{6\left(\theta^{2}+4 \alpha\right)}{\theta^{3}\left(\theta^{2}+\alpha\right)}
$$




$$
\mu_{4}^{\prime}=\frac{\theta^{2}+2 \alpha}{\theta\left(\theta^{2}+\alpha\right)}+\frac{14\left(\theta^{2}+3 \alpha\right)}{\theta^{2}\left(\theta^{2}+\alpha\right)}+\frac{36\left(\theta^{2}+4 \alpha\right)}{\theta^{3}\left(\theta^{2}+\alpha\right)}+\frac{24\left(\theta^{2}+5 \alpha\right)}{\theta^{4}\left(\theta^{2}+\alpha\right)}
$$

By using the identity $\mu_{r}=E(X-\mu)^{r}=\sum_{k=0}^{r}\left(\begin{array}{l}r \\ k\end{array}\right) \mu_{k}^{\prime}\left(-\mu_{1}^{\prime}\right)^{r-k}$, the central moments can be obtained as follows:

$$
\begin{gathered}
\mu_{2}=\sigma^{2}=\frac{\theta^{5}+\theta^{4}+3 \theta^{3} \alpha+4 \theta^{2} \alpha+2 \theta \alpha^{2}+2 \alpha^{2}}{\theta^{2}\left(\theta^{2}+\alpha\right)^{2}} \\
\mu_{3}=\frac{2 \alpha^{3}(\theta+1)(\theta+2)+\alpha^{2}\left(5 \theta^{4}+18 \theta^{3}+12 \theta^{2}\right)+\alpha\left(4 \theta^{6}+15 \theta^{5}+12 \theta^{4}\right)+\theta^{6}(\theta+1)(\theta+2)}{\theta^{3}\left(\theta^{2}+\alpha\right)^{3}} \\
\mu_{4}=\frac{\alpha^{4}\left(2 \theta^{3}-4 \theta^{2}+48 \theta+216\right)+\alpha^{3}\left(7 \theta^{5}+40 \theta^{4}+180 \theta^{3}+624 \theta^{2}\right)+}{\left(\theta^{11}+4 \theta^{6}+240 \theta^{5}+476 \theta^{4}-336 \theta^{2}\right)+\alpha\left(5 \theta^{9}+126 \theta^{7}+264 \theta^{6}-72 \theta^{4}-96 \theta^{2}\right)+} \\
\left.\theta^{4}\left(\theta^{2}+\alpha\right)^{4}\right)
\end{gathered}
$$

Some important indices of the shape of the distribution are skewness, kurtosis, index of dispersion and the coefficient of variation respectively. These measures are given by:

Skewness:

$$
\left(\sqrt{\left.\beta_{1}\right)}=\frac{\mu_{3}}{\left(\mu_{2}\right)^{\frac{3}{2}}}=\frac{(\theta+1)(\theta+2)\left\{2 \alpha^{3}+\theta^{6}\right\}+\alpha^{2}\left(5 \theta^{4}+18 \theta^{3}+12 \theta^{2}\right)+\alpha\left(4 \theta^{6}+15 \theta^{5}+12 \theta^{4}\right)}{\left(\theta^{5}+\theta^{4}+3 \theta^{3} \alpha+4 \theta^{2} \alpha+2 \alpha^{2}(\theta+1)\right)^{3 / 2}} .\right.
$$

Kurtosis:

$$
\left(\beta_{2}\right)=\frac{\mu_{4}}{\sigma^{4}}=\frac{A \alpha^{4}+B \alpha^{3}+C \alpha^{2}+D \alpha+E}{\left(\theta^{5}+\theta^{4}+3 \theta^{3} \alpha+4 \theta^{2} \alpha+2 \theta \alpha^{2}+2 \alpha^{2}\right)^{2}}
$$

where,

$$
\begin{gathered}
A=2 \theta^{3}+26 \theta^{2}+48 \theta+24 \\
B=7 \theta^{5}+92 \theta^{4}+180 \theta^{3}+96 \theta^{2} \\
C=9 \theta^{7}+116 \theta^{6}+240 \theta^{5}+132 \theta^{4} \\
D=5 \theta^{9}+60 \theta^{8}+126 \theta^{7}+72 \theta^{6} \\
E=\theta^{11}+10 \theta^{10}+18 \theta^{9}+9 \theta^{8}
\end{gathered}
$$

\subsection{Index of Dispersion (ID) and Coefficient of Variation (CV)}

The index of dispersion (ID) (Johnson et al., [8, p. 163]) is defined as the ratio of variance and mean is useful in determining whether a distribution is over dispersed or under dispersed for ID $>(<) 1$.

Since the index of dispersion given as

$$
\gamma=\frac{\sigma^{2}}{\mu}=1+\frac{\theta^{4}+4 \theta^{2} \alpha+2 \alpha^{2}}{\theta\left(\theta^{2}+\alpha\right)\left(\theta^{2}+2 \alpha\right)}>1
$$

for $\theta>0$ and fixed $\alpha$.Therefore it follows that the $N Q P L(\alpha, \theta)$ distribution is over-dispersed.

Coefficient of variation is defined as the ratio of standard deviation to mean and is given as

$$
C . V=\frac{\sqrt{2 \alpha^{2}(\theta+1)+\alpha\left(3 \theta^{3}+4 \theta^{2}\right)+\theta^{5}+\theta^{4}}}{\theta^{2}+2 \alpha} \text {. }
$$




\section{Remarks:}

(i) $\sqrt{\beta_{1}}$ is an increasing function of $\theta$ for fixed $\alpha$ and $\frac{3}{\sqrt{\alpha+2}}<\sqrt{\beta_{1}}<\infty$.

(ii) $\beta_{2}$ is an increasing function in $\theta$ for fixed $\alpha$ and $\frac{3(\alpha+3)}{\alpha+1}<\beta_{2}<\infty$

(iii) C.V is an increasing function in $\theta$ for fixed $\alpha$ and $\frac{1}{\sqrt{\alpha+1}}<C$.V $<\infty$.

\section{Probability generating function}

The probability generating function of new quasi Poisson Lindley (NQPL) distribution is given as

$$
\begin{gathered}
G(t)=E\left(t^{x}\right)=\sum_{x=0}^{\infty} t^{x} P(x) \\
=\frac{\theta^{2}}{\left(\theta^{2}+\alpha\right)(\theta+1-t)^{2}}\{\theta(\theta+1-t)+\alpha\}
\end{gathered}
$$

The expression for recurrence relation for probabilities obtained from (4.1) is

where,

$$
p_{r}=\frac{1}{(\theta+1)^{2}}\left\{2(\theta+1) p_{r-1}-p_{r-2}\right\}, \quad r>1
$$

$$
\begin{aligned}
& p_{0}=\left(\frac{\theta}{\theta+1}\right)^{2}\left\{\frac{\theta(\theta+1)+\alpha}{\theta^{2}+\alpha}\right\} \\
& p_{1}=\frac{\theta^{2}}{(\theta+1)^{3}}\left\{\frac{\theta(\theta+1)+2 \alpha}{\theta^{2}+\alpha}\right\}
\end{aligned}
$$

The $r^{t h}$ order probability of NQPL distribution may also be written as

$$
p_{r}=\frac{\theta^{2}}{(1+\theta)^{r}}\left\{\frac{\theta(\theta+1)+r \alpha}{\theta^{2}+\alpha}\right\}
$$

\section{Size-biased Version of New Quasi Poisson -Lindley distribution}

Mostly, when an observation is recorded according to certain stochastic model, the distribution of recorded observation may differ, unless equal probability of recording the observations is being employed. Then the observed distribution that arises will have density of the form

$$
f_{w}(x)=\frac{w(x) f(x)}{E[w(x)]}
$$

where, $f(x)$ is the p.m.f of the original distribution, $w(x)$ is the weighted function $(w(x)>0)$ and $E[w(x)]$ is the mean of the original distribution. These models were introduced by Rao (1965) and called them as weighted distribution. The distribution with weight $w(x)=x$ is called as the size-biased distribution.

Preposition. Let $X$ is a random variable with $N Q P L(\alpha, \theta)$ distribution. The probability mass function (pmf) and probability generating function (pgf) of size biased new quasi Poisson-Lindley distribution may be given as 


$$
f_{S}(x ; \theta, \alpha)=\frac{\theta^{3} x}{\left(\theta^{2}+2 \alpha\right)(1+\theta)^{x+1}}\left[\theta+\frac{\alpha(x+1)}{(1+\theta)}\right], \quad x=1,2, \ldots,
$$

and

$$
G_{S}(t)=\frac{\theta^{3} t}{\left(\theta^{2}+2 \alpha\right)(1+\theta-t)^{3}}[\theta(\theta+1-t)+2 \alpha]
$$

\section{Recurrence Relations for Probabilities SBNQPL distribution}

The expression for recurrence relation for probabilities of SBNQPL distribution may be given as

$$
p_{r}=\frac{1}{(\theta+1)^{3}}\left\{3(\theta+1)^{2} p_{r-1}-3(\theta+1) p_{r-2}+p_{r-3}\right\}, \quad r>3
$$

where

$$
p_{1}=\frac{\theta^{3}\left(\theta^{2}+\theta+2 \alpha\right)}{(1+\theta)^{3}\left(\theta^{2}+2 \alpha\right)}, \quad p_{2}=\frac{2 \theta^{3}\left(\theta^{2}+\theta+3 \alpha\right)}{(1+\theta)^{4}\left(\theta^{2}+2 \alpha\right)}, \text { and } p_{3}=\frac{3 \theta^{3}\left(\theta^{2}+\theta+4 \alpha\right)}{(1+\theta)^{5}\left(\theta^{2}+2 \alpha\right)}
$$

\section{Estimation of Parameters of NQPL distribution}

\subsection{Method of Moments}

The first two moments are required to estimate the parameters by the method of moments.

$$
\frac{\mu_{2}^{\prime}-\mu_{1}^{\prime}}{\mu_{1}^{\prime 2}}=\frac{2\left(\theta^{2}+3 \alpha\right)\left(\theta^{2}+\alpha\right)}{\left(\theta^{2}+2 \alpha\right)^{2}}=k \text { (say) }
$$

Substituting $\alpha=b \theta^{2}$, in (7.1) a quadratic equation in ' $b$ ' is obtained as

$$
(6-4 k) b^{2}+4(2-k) b+(2-k)=0
$$

Replacing the first two population moment by the respective sample moments in (6.1) the estimate of k may be obtained. Substituting the estimate of $\mathrm{k}$ in (6.2) the estimate $\hat{b}$ can be obtained from the quadratic equation (7.2). We have,

$$
\bar{X}=\frac{\theta^{2}+2 \alpha}{\theta\left(\theta^{2}+\alpha\right)}
$$

Substituting $\alpha=b \theta^{2}$ in the expression (7.1.3) we have

$$
\bar{X}=\frac{1+2 b}{\theta(1+b)} .
$$

Hence

$$
\hat{\theta}=\left(\frac{1+2 b}{1+b}\right) \frac{1}{\bar{X}},
$$

and

$$
\hat{\alpha}=b \hat{\theta}^{2}=\frac{b(1+2 b)^{2}}{(1+b)^{2}(\bar{X})^{2}} .
$$




\subsection{Maximum Likelihood Estimates}

Let $x_{1}, x_{2}, \ldots, x_{n}$ be a random sample of size $\mathrm{n}$ from the NQPL distribution (2.1) and let $f_{x}$ be the observed frequency in the sample corresponding to $X=x(x=1,2, \ldots, k)$ such that $\sum_{x=1}^{k} f_{x}=n$.

The Likelihood function $L$ of the new quasi Poisson Lindley distribution is given as

$$
L=\prod_{i=1}^{k} f\left(x_{i} ; \theta, \alpha\right)=\left(\frac{\theta^{2}}{\theta^{2}+\alpha}\right)^{n} \frac{1}{(1+\theta)^{\sum_{i=1}^{k}(x+2) f_{x}}} \prod_{i=1}^{n}[\theta(\theta+1)+\alpha(x+1)]^{f_{x}} .
$$

The log likelihood function becomes

$$
\log L=n \log \left(\frac{\theta^{2}}{\theta^{2}+\alpha}\right)-\sum_{x=1}^{k} f_{x}(x+2) \log (1+\theta)+\sum_{x=1}^{k} f_{x} \log [\theta(\theta+1)+\alpha(x+1)]
$$

Then the log likelihood equations are obtained as

$$
\frac{\partial \log L}{\partial \alpha}=-\frac{n}{\theta^{2}+\alpha}+\sum_{x=1}^{k} \frac{(x+1) f_{x}}{[\theta(\theta+1)+\alpha(x+1)]}=0
$$

and

$$
\frac{\partial \log L}{\partial \theta}=\frac{2 n}{\theta}-\frac{2 n}{\theta^{2}+\alpha}-\sum_{x=1}^{k} \frac{(x+2) f_{x}}{1+\theta}+\sum_{x=1}^{k} \frac{(2 \theta+1) f_{x}}{[\theta(\theta+1)+\alpha(x+1)]}=0
$$

The above two equations could be solved using Fisher's scoring method. We have

$$
\begin{gathered}
\frac{\partial^{2} \log L}{\partial \alpha^{2}}=\frac{n}{\left(\theta^{2}+\alpha\right)^{2}}-\sum_{x=1}^{k} \frac{(x+1)^{2} f_{x}}{[\theta(\theta+1)+\alpha(x+1)]^{2}} \\
\frac{\partial^{2} \log L}{\partial \alpha \partial \theta}=\frac{2 n \theta}{\left(\theta^{2}+\alpha\right)^{2}}-\sum_{x=1}^{k} \frac{(x+1)(2 \theta+1) f_{x}}{[\theta(\theta+1)+\alpha(x+1)]^{2}} \\
\frac{\partial^{2} \log L}{\partial \theta^{2}}=-\frac{2 n}{\theta^{2}}+\frac{4 n \theta}{\left(\theta^{2}+\alpha\right)^{2}}+\sum_{x=1}^{k} \frac{(x+2) f_{x}}{(1+\theta)^{2}}+\sum_{x=1}^{k} \frac{\left(2 \theta^{2}+2 \theta+1-2 \alpha(x+1)\right) f_{x}}{[\theta(\theta+1)+\alpha(x+1)]^{2}} .
\end{gathered}
$$

The following system equations can be solved for $\hat{\theta}$ and $\hat{\alpha}$ iteratively till sufficiently close values of $\hat{\theta}$ and $\hat{\alpha}$ are obtained, where $\theta_{0}$ and $\alpha_{0}$ are the initials value of $\theta$ and $\alpha$ respectively.

$$
\left[\begin{array}{ll}
\frac{\partial^{2} \log L}{\partial \alpha^{2}} & \frac{\partial^{2} \log L}{\partial \alpha \partial \theta} \\
\frac{\partial^{2} \log L}{\partial \alpha \partial \theta} & \frac{\partial^{2} \log L}{\partial \theta^{2}}
\end{array}\right]\left[\begin{array}{l}
\hat{\theta}-\theta_{0} \\
\hat{\alpha}-\alpha_{0}
\end{array}\right]=\left[\begin{array}{c}
\frac{\partial \log L}{\partial \alpha} \\
\frac{\partial \log L}{\partial \theta}
\end{array}\right]_{\substack{\hat{\theta}=\theta_{0} \\
\hat{\alpha}=\alpha_{0}}} .
$$




\section{Estimation of parameters of SBNQPL distribution}

\subsection{Method of moments}

The first two moments are required to estimate the parameters by the method of moments.

$$
\frac{\left(\mu^{\prime \prime}{ }_{2}-1\right)-3\left(\mu_{1}^{\prime}-1\right)}{\left(\mu_{1}^{\prime}-1\right)^{2}}=\frac{3\left(\theta^{2}+4 \alpha\right)\left(\theta^{2}+2 \alpha\right)}{2\left(\theta^{2}+3 \alpha\right)^{2}}=k \text { (say) }
$$

Substituting $\alpha=b \theta^{2}$, in (8.1.1) a quadratic equation in ' $b$ ' is obtained as

$$
(24-18 k) b^{2}+6(3-2 k) b+(3-2 k)=0
$$

Replacing the first two population moment by the respective sample moments in (8.1) the estimate of k may be obtained. Substituting the estimate of $\mathrm{k}$ in (8.2) the estimate $\hat{b}$ can be obtained from the quadratic equation (8.2). The mean of SBNQPL distribution may be given as

$$
\bar{X}=1+\frac{2\left(\theta^{2}+3 \alpha\right)}{\theta\left(\theta^{2}+2 \alpha\right)}
$$

Substituting $\alpha=b \theta^{2}$, in the above expression (8.3) we have

$$
\hat{\theta}=\frac{2(1+3 b)}{(1+2 b)(\bar{X}-1)}
$$

and

$$
\hat{\alpha}=b \hat{\theta}^{2}=\frac{4 b(1+3 b)^{2}}{(1+2 b)^{2}(\bar{X}-1)^{2}}
$$

where

$$
\bar{X}-1=\frac{2(1+3 b)}{\theta(1+2 b)}
$$

\subsection{Maximum Likelihood Estimates}

Let $x_{1}, x_{2}, \ldots \ldots, x_{n}$ be a random sample of size $\mathrm{n}$ from the SBNQPL distribution and let $f_{x}$ be the observed frequency in the sample corresponding to $X=x(x=1,2, \ldots \ldots, k)$ such that $\sum_{x=1}^{k} f_{x}=n$. The Likelihood function $L$ of the new quasi Poisson Lindley distribution is given as

$$
L=\prod_{i=1}^{k} f\left(x_{i} ; \theta, \alpha\right)=\left(\frac{\theta^{3}}{\theta^{2}+\alpha}\right)^{n} \frac{1}{(1+\theta)^{\sum_{i=1}^{k}(x+2) f_{x}}} \prod_{i=1}^{n}\left[\alpha x^{2}+x\left(\theta^{2}+\theta+\alpha\right)\right]^{f_{x}}
$$

The log likelihood function becomes

$$
\log L=n \log \left(\frac{\theta^{3}}{\theta^{2}+\alpha}\right)-\sum_{x=1}^{k} f_{x}(x+2) \log (1+\theta)+\sum_{x=1}^{k} f_{x} \log \left[\alpha x^{2}+x\left(\theta^{2}+\theta+\alpha\right)\right]
$$


Then the log likelihood equations are obtained as

$$
\frac{\partial \log L}{\partial \alpha}=-\frac{n}{\theta^{2}+\alpha}+\sum_{x=1}^{k} \frac{\left(x^{2}+x\right) f_{x}}{\left[\alpha x^{2}+x\left(\theta^{2}+\theta+\alpha\right)\right]}=0
$$

and

$$
\frac{\partial \log L}{\partial \theta}=\frac{3 n}{\theta}-\frac{2 n \theta}{\theta^{2}+\alpha}-\sum_{x=1}^{k} \frac{(x+2) f_{x}}{1+\theta}+\sum_{x=1}^{k} \frac{x(2 \theta+1) f_{x}}{\left[\alpha x^{2}+x\left(\theta^{2}+\theta+\alpha\right)\right]}=0 .
$$

The above two equations could be solved using Fisher's scoring method. We have

$$
\begin{gathered}
\frac{\partial^{2} \log \mathrm{L}}{\partial \alpha^{2}}=\frac{n}{\left(\theta^{2}+\alpha\right)^{2}}-\sum_{x=1}^{k} \frac{\left(x^{2}+x\right)^{2} f_{x}}{\left[\alpha x^{2}+x\left(\theta^{2}+\theta+\alpha\right)\right]^{2}}, \\
\frac{\partial^{2} \log \mathrm{L}}{\partial \alpha \partial \theta}=\frac{2 n \theta}{\left(\theta^{2}+\alpha\right)^{2}}-\sum_{x=1}^{k} \frac{x\left(x^{2}+x\right)(2 \theta+1) f_{x}}{\left[\alpha x^{2}+x\left(\theta^{2}+\theta+\alpha\right)\right]^{2}}, \\
\frac{\partial^{2} \log \mathrm{L}}{\partial \theta^{2}}=-\frac{3 n}{\theta^{2}}+\frac{4 n \theta}{\left(\theta^{2}+\alpha\right)^{2}}+\sum_{x=1}^{k} \frac{(x+2) f_{x}}{(1+\theta)^{2}}+\sum_{x=1}^{k} \frac{\left(2 \alpha x^{2}-2 x \theta^{2}-2 \theta x+2 \alpha x-1\right) f_{x}}{\left[\alpha x^{2}+x\left(\theta^{2}+\theta+\alpha\right)\right]^{2}} .
\end{gathered}
$$

The following equations can be solved for $\hat{\theta}$ and $\hat{\alpha}$ iteratively till sufficiently close values of $\hat{\theta}$ and $\hat{\alpha}$

$$
\left[\begin{array}{ll}
\frac{\partial^{2} \log \mathrm{L}}{\partial \alpha^{2}} & \frac{\partial^{2} \log \mathrm{L}}{\partial \alpha \partial \theta} \\
\frac{\partial^{2} \log \mathrm{L}}{\partial \alpha \partial \theta} & \frac{\partial^{2} \log \mathrm{L}}{\partial \theta^{2}}
\end{array}\right]\left[\begin{array}{c}
\hat{\theta}-\theta_{0} \\
\hat{\alpha}-\alpha_{0}
\end{array}\right]=\left[\begin{array}{c}
\frac{\partial \log L}{\partial \alpha} \\
\frac{\partial \log L}{\partial \theta}
\end{array}\right]_{\substack{\hat{\theta}=\theta_{0} \\
\hat{\alpha}=\alpha_{0}}}
$$

where $\theta_{0}$ and $\alpha_{0}$ are the initials value of $\theta$ and $\alpha$ respectively.

\section{Goodness of fit}

The fittings of the two-parameter NQPL distribution based on two data-sets have been presented in the Tables 1 and 2. The expected frequencies according to the one parameter Poisson-Lindley with parameter $\theta$, two parameter Poisson-Lindley distributions with parameter $\theta$ and $\alpha$ in Table 1 presented by Shanker et ai. [11] and two parameter discrete gamma with parameter $\mathrm{k}$ and $\theta$ in Table 2 presented by Chakraborty and Chakravarty [13] have also been given for ready comparison with NQPL distribution. The estimates of the parameters have been obtained by the method of moments.

The SBNQPL distribution has also been fitted to three sets of data reported by James (1953), Coleman and James (1961), and Simonoff (2003). The expected frequencies of SBNQPL distribution along with SBPL and SBQPL distributions have been shown in Tables 3,4 and 5 for its comparison. The estimates of the parameters have been obtained by the method of moments. However, better estimates may be obtained by using the method of maximum likelihood. It is observed that to all three data sets, the SBNQPL provides closer fit than SBPL distribution. 
Table 1. Observed and expected frequencies for distribution of Pyrausta nublilalis in 1937.

\begin{tabular}{|c|l|l|l|l|}
\hline \multirow{2}{*}{$\begin{array}{l}\text { No. of } \\
\text { accidents }\end{array}$} & $\begin{array}{l}\text { Observed } \\
\text { frequencies }\end{array}$ & \multicolumn{3}{|c|}{ Expected frequencies } \\
\cline { 3 - 5 } & & $\begin{array}{l}\text { Poisson- } \\
\text { Lindley }(\theta)\end{array}$ & $\begin{array}{l}\text { Poisson- } \\
\text { Lindley }(\theta, \alpha)\end{array}$ & NQPL $(\alpha, \theta)$ \\
\hline 0 & 33 & 31.49 & 31.9 & 31.90 \\
1 & 12 & 14.16 & 13.8 & 13.80 \\
2 & 6 & 6.09 & 5.9 & 5.92 \\
3 & 3 & 2.54 & 2.5 & 2.73 \\
4 & 1 & 1.04 & 1.1 & 1.09 \\
$\geq 5$ & 1 & 0.42 & 0.8 & 0.56 \\
\hline \multicolumn{2}{|c}{56} & 56 & 56 & 56 \\
& & $\hat{\theta}=1.8082$ & $\hat{\alpha}=0.2573$ & $\hat{\alpha}=0.3920$ \\
& & & $\hat{\theta}=0.39249$ & $\hat{\theta}=1.5255$ \\
& \multirow{2}{*}{$\chi^{2}$} & 4.82 & 0.36 & 0.3203 \\
& $P$ value & 0.1855 & 0.8353 & 0.852 \\
\hline
\end{tabular}

Table 2. Distribution of number of European red mites on apple leaves

\begin{tabular}{|l|l|l|l|l|}
\hline \multirow{2}{*}{$\begin{array}{l}\text { European red } \\
\text { mites }\end{array}$} & \multirow{2}{*}{$\begin{array}{l}\text { Observed } \\
\text { frequencies }\end{array}$} & \multicolumn{3}{|c|}{ Expected frequencies } \\
\cline { 3 - 5 } & & $d \gamma(k, \theta)$ & $\mathrm{NBD}(\mathrm{r}, \mathrm{p})$ & $\mathrm{NQPL}(\alpha, \theta)$ \\
\hline 0 & 70 & 69.67 & 69.49 & 67.75 \\
1 & 38 & 37.49 & 37.6 & 38.51 \\
2 & 17 & 20.02 & 20.1 & 20.99 \\
3 & 10 & 10.67 & 10.7 & 11.11 \\
4 & 9 & 5.69 & 5.69 & 5.77 \\
5 & 3 & 3.03 & 3.02 & 3.14 \\
6 & 2 & 1.61 & 1.6 & 1.68 \\
7 & 1 & .86 & 0.85 & .84 \\
8 & 0 & .96 & 0.95 & .30 \\
\hline \multicolumn{2}{|c|}{150} & 150 & 150 & 150 \\
& & $\hat{k}=1.0078$ & $\hat{r}=1.0245$ & $\hat{\alpha}=0.9121$ \\
\multicolumn{2}{|c}{$\chi^{2}$} & $\hat{\theta}=1.5830$ & $\hat{p}=0.5281$ & $\hat{\theta}=1.2076$ \\
& P value & 2.89 & 2.91 & 2.7592 \\
\hline
\end{tabular}

The fitting of two parameter NQPL distribution along with $\chi^{2}$ and p-values has been presented to two sets of data. From the above Tables it is observed that NQPL distribution provides closer fits. 
Table 3. Fitting of SBNQPL distribution for the Counts of people in public places on a spring afternoon in Portland.

\begin{tabular}{|c|c|c|c|c|}
\hline \multirow[t]{2}{*}{ Size of Groups } & \multirow{2}{*}{$\begin{array}{l}\text { Observed } \\
\text { frequencies }\end{array}$} & \multicolumn{3}{|c|}{ Expected frequencies } \\
\hline & & $\operatorname{SBPL}(\theta)$ & $\operatorname{SBQPL}(\theta, \alpha)$ & SBNQPL $(\alpha, \theta)$ \\
\hline 1 & 1486 & 1532.5 & 1485.4 & 1484.00 \\
\hline 2 & 694 & 630.6 & 697.3 & 699.41 \\
\hline 3 & 195 & 191.9 & 189.7 & 191.73 \\
\hline 4 & 37 & 51.3 & 40.6 & 38.78 \\
\hline 5 & 10 & 12.8 & 8.3 & 7.73 \\
\hline 6 & 1 & 3.9 & 1.7 & 1.35 \\
\hline & $\begin{array}{l}\chi^{2} \\
P \text { value }\end{array}$ & $\begin{array}{l}2423 \\
\hat{\theta}=4.5082 \\
13.766 \\
0.01\end{array}$ & $\begin{array}{l}2423 \\
\hat{\alpha}=-0.79159 \\
\hat{\theta}=7.14227 \\
0.710 \\
0.70\end{array}$ & $\begin{array}{l}2423 \\
\hat{\alpha}=-63.6352 \\
\hat{\theta}=7.2136 \\
0.588 \\
0.745\end{array}$ \\
\hline
\end{tabular}

Table 4. Fitting of SBNQPL distribution for the Counts of Shopping Groups-Eugene, Spring, Department Store and Public Market.

\begin{tabular}{|c|l|l|l|l|}
\hline Size of Groups & \multirow{2}{*}{$\begin{array}{l}\text { Observed } \\
\text { frequencies }\end{array}$} & \multicolumn{3}{|c|}{ Expected frequencies } \\
\cline { 3 - 5 } & & SBPL $(\theta)$ & SBQPL $(\theta, \alpha)$ & SBNQPL $(\alpha, \theta)$ \\
\hline 1 & 316 & 323.0 & 315.7 & 315.52 \\
2 & 141 & 132.5 & 142.9 & 143.07 \\
3 & 44 & 40.2 & 40.5 & 42.48 \\
4 & 5 & 10.7 & 8.9 & 7.10 \\
5 & 4 & 3.6 & 2.0 & 1.84 \\
\hline & 510 & 510.0 & 510.0 & 510.0 \\
& & $\hat{\theta}=4.5224$ & $\hat{\alpha}=-0.52229$ & $\hat{\alpha}=-81.0372$ \\
& & & $\hat{\theta}=6.57872$ & $\hat{\theta}=6.6080$ \\
& \multirow{2}{*}{$\chi^{2}$} & 3.021 & 0.659 & 0.0854 \\
& $P$ value & 0.40 & 0.44 & 0.77 \\
\hline
\end{tabular}

Table 5. Fitting of SBNQPL distribution for Counts of Play Groups-Eugene, Spring, Public Playground D.

\begin{tabular}{|c|c|c|c|c|}
\hline \multirow{2}{*}{$\begin{array}{l}\text { No. of } \\
\text { accidents }\end{array}$} & \multirow{2}{*}{$\begin{array}{l}\text { Observed } \\
\text { frequencies }\end{array}$} & \multicolumn{3}{|c|}{ Expected frequencies } \\
\hline & & $\operatorname{SBPL}(\theta)$ & $\operatorname{SBQPL}(\theta, \alpha)$ & $\operatorname{SBNQPL}(\alpha, \theta)$ \\
\hline 1 & 305 & 314.4 & 304.2 & 305.61 \\
\hline 2 & 144 & 134.4 & 148.5 & 146.41 \\
\hline 3 & 50 & 42.5 & 42.8 & 44.58 \\
\hline 4 & 5 & 11.8 & 9.2 & 7.94 \\
\hline 5 & 2 & 3.1 & 1.9 & 2.06 \\
\hline 6 & 1 & 0.8 & 0.4 & 0.40 \\
\hline & $\begin{array}{l}\chi^{2} \\
P \text { value }\end{array}$ & $\begin{array}{l}507.0 \\
\hat{\theta}=4.3179 \\
6.415 \\
0.043\end{array}$ & $\begin{array}{l}507.0 \\
\hat{\alpha}=-0.76547 \\
\hat{\theta}=6.74857 \\
2.415 \\
0.130\end{array}$ & $\begin{array}{l}507.0 \\
\hat{\alpha}=-66.4950 \\
\hat{\theta}=6.4346 \\
1.2536 \\
0.263\end{array}$ \\
\hline
\end{tabular}




\section{Conclusion}

In this paper, we propose a two-parameter new Quasi Poisson-Lindley (NQPL) distribution, of which the oneparameter PL is a particular case. Several properties of the new NQPL distribution such as moments, skewness and Kurtosis have been discussed. It has been shown that the NQPL distribution is more flexible than PL distribution. The indexes of dispersion and coefficient of variation of the NQPL distribution have been discussed. The parameters for both the NQPL and SBNQPL distributions have been estimated by using the method of moments and the method of maximum likelihood. Finally, both the NQPL and SBNQPL distributions have been fitted to a few sets of data for testing its goodness of fit. It is found that both the distributions provide closer fits.

\section{References}

[1] S. Chakraborty and D. Chakravarty, Discrete Gamma distributions: Properties and Parameter Estimations, Communication in Statistics-Theory and Methods, 41 (2012), 3301-3324.

[2] J. S. Coleman and J. James, The equilibrium size distribution of freely forming groups, Sociometry, 24 (1961), 36-45.

[3] E. G. Deniz and E. C. Ojeda, The discrete Lindley distribution - Properties and Applications, Journal of Statistical Computation and Simulation, 81(11) (2011), 1405-1416.

[4] M. E. Ghitany, B. Atieh, S. Nadarajah, Lindley distribution and its Applications, Mathematics and ComputersinSimulation, 78(4) (2008), 493-506.

[5] M. E. Ghitany, D. K. Al-Mutairi, S. Nadarajah, Zero-truncated Poisson-Lindley distribution and its Applications, Mathematics and Computers in Simulation,79(3) (2008), 279-287.

[6] M. E. Ghitany and D. K.. Al-Mutairi, Size-biased Poisson-Lindley distribution and its Applications, Metron - International Journal of Statistics, LXVI(3) (2008), 299-311.

[7] M. E. Ghitany and D. K. Al-Mutairi, Estimation methods for the discrete Poisson-Lindley distribution, Journal of Statistical Computation and Simulation, 79(1) (2009), 1-9.

[8] N. L. Johnson, A. W. Kemp and S. Kotz, Univariate Discrete Distributions (Hoboken, NJ: John Wiley \& Sons, 2005).

[9] D. V. Lindley, Fiducial distributions and Bayes' theorem, Journal of the Royal Statistical Society, Series B, 20 (1958), $102-107$.

[10] M. Sankaran, The discrete Poisson-Lindley distribution, Biometrics, 26 (1970), 145-149.

[11] R. Shanker and A. Mishra, A Quasi Lindley Distribution, African Journal of Mathematics and Computer Science Research, 6(4) (2013), 64-71.

[12] R. Shanker and A. Mishra, On a Size-Biased Quasi Poisson-Lindley Distribution, International Journal of Probability and Statistics, 2(2) (2013), 28-34.

[13] R. Shanker and A. H. Ghebretsadik, A New Quasi Lindley Distribution, International Journal of Statistics and Systems, 8(2) (2013), 143-156. 